\title{
Applications cliniques de la superoxyde dismutase dans les fibroses cutanées radioinduites
}

B. PERDEREAU ${ }^{\star}$, F. CAMPANA**, R.J. VILCOQ**, D. PONTVERT ${ }^{\star \star}$, A. FOURQUET ${ }^{\star *}$, C. BARBAROUX* ${ }^{*}$, H. MAGDELENAT* *Service de Radiopathologie, "*Service de Radiothérapie, Institut Curie, 26 rue d'Ulm, 75005 Paris

Une étude portant sur l'évaluation de l'effet thérapeutique de la SOD sous forme de pommade sur les radiofibroses cutanées pour cancer du sein a porté sur 75 patientes dont 19 ont permis aux auteurs de rechercher à travers la relation dose / effet la posologie la mieux adaptée (800 U 2 fois/jour pendant 90 jours). Le protocole de contrôle des effets thérapeutiques comprend, outre l'observation clinique minutieuse, des mesures quantifiées : téléthermographie I.R., pH-métrie cutanée, planimétrie dont l'exploitation conduit à proposer un score analytique tenant compte du retentissement loco-régional. Les résultats font tous état d'une incontestable amélioration d'autant plus marquée que l'apparition de la fibrose est plus récente ; elle est en moyenne de $32 \%$ du score initial mais peut atteindre $50 \%$. La chronologie des phases de récupération a pu être dégagée :

1) diminution voire cessation de la douleur dès la $3-4^{e}$ semaine,

2) assouplissement associé à une réduction du placard fibreux à partir des 2 et $3^{e}$ mois,

3) affaiblissement discret de la pigmentation après le $4^{\mathrm{e}}$ mois,

4) enfin, une remobilisation du membre supérieur tardive (> 12 mois) après le début du traitement. 